\title{
Mucoepidermoid carcinoma of the sublingual gland harboring a translocation of the $M A M L 2$ gene: A case report
}

\author{
KIMINOBU SATO $^{1,2}$, JUN AKIBA ${ }^{3}$, KEN NAKAMURA $^{2}$, HIDEYUKI ABE $^{3}$, AKIHIKO KAWAHARA $^{3}$, \\ TAKEICHIRO ASO ${ }^{1}$, HIROHITO UMENO ${ }^{1}$, HIROSHI HARADA ${ }^{4}$ and HIROHISA YANO ${ }^{2}$
}

\author{
Departments of ${ }^{1}$ Otolaryngology-Head and Neck Surgery and ${ }^{2}$ Pathology, Kurume University School of Medicine; \\ ${ }^{3}$ Department of Diagnostic Pathology, Kurume University Hospital, Kurume, Fukuoka 830-0011; ${ }^{4}$ Department of \\ Diagnostic Pathology, Seichokai Fuchu Hospital, Izumi, Osaka 594-0076, Japan
}

Received January 16, 2017; Accepted May 8, 2017

DOI: $10.3892 / 01.2017 .6550$

\begin{abstract}
Among tumors of the major salivary glands, tumors in the sublingual gland are rare. Although mucoepidermoid carcinoma (MEC) represents a histological type of salivary gland tumor, it is occasionally difficult to diagnose due to its histological variation. The present study reports a case of MEC harboring a mastermind-like transcriptional coactivator 2 (MAML2) gene translocation in the sublingual gland. A 76-year-old Japanese woman with a mass in the left submandibular region was referred to Kurume University Hospital (Kurume, Japan). Computed tomography scans revealed that the tumor was predominantly located in the sublingual gland, and tumor resection was performed. Histologically, the tumor was composed of cells that exhibited low-grade nuclear atypia and clear and/or granular eosinophilic cytoplasm, and that were proliferating in solid patterns. Periodic acid-Schiff and alcian blue staining revealed a small number of mucinous cells in the tumor. Immunohistochemically, the tumor cells were positive for p40 and p63. Fluorescence in situ hybridization (FISH) analysis revealed a MAML2 gene split. The definitive pathological diagnosis was low-grade MEC, as the case lacked any factors indicative of high-grade malignancy. To the best of our knowledge, this is the first report of MEC in the sublingual gland with MAML2 gene translocation confirmed by FISH.
\end{abstract}

\section{Introduction}

Among tumors of the major salivary glands, tumors of the sublingual gland are rare, accounting for $<0.5 \%$ of cases (1). Although mucoepidermoid carcinoma (MEC) is one of the

Correspondence to: Dr Kiminobu Sato, Department of Otolaryngology-Head and Neck Surgery, Kurume University School of Medicine, 67 Asahi-machi, Kurume, Fukuoka 830-0011, Japan

E-mail: satou_kiminobu@med.kurume-u.ac.jp

Key words: mucoepidermoid carcinoma, sublingual gland, major salivary glands, fluorescence in situ hybridization, MAML2 gene split most common malignant salivary gland neoplasms, and $\sim 50 \%$ of MECs occur in the major salivary glands, including the parotid or submandibular gland $(2,3)$, MEC of the sublingual gland is a relatively rare disease.

MEC is a malignant epithelial neoplasm that was first described by Stewart et al in 1945 (4). MEC is the most common malignant salivary gland tumor among cases reviewed at the Armed Forces Institute of Pathology since 1970 and other treatment centers in the United States (2). In addition, the frequencies are comparable to those studies from other countries (2).

MEC is histologically characterized by the presence of mucinous, intermediate and squamoid cells, while clear, columnar and oncocytic cells are less commonly observed $(3,5)$. Typically, the pathological diagnosis of MEC is relatively easy; however, definitive diagnosis may be challenging when the tumor is composed of less common cell types (5). MEC is sub-classified into low, intermediate and high grades on the basis of its histological features $(2,3,5,6)$.

No chemical carcinogens or oncogenic viruses are associated with MEC (6). However, prior exposure to radiation is a contributing factor for MEC (7).

Prognosis depends on clinical stage, site, grading and adequacy of surgical excision (5). Low grade tumors exhibit excellent prognoses, however; high grade tumors demonstrate metastasis to the regional lymph nodes and distant sites including lungs, bone, and brain (5).

Mastermind-like transcriptional coactivator 2 (MAML2) gene translocation is observed in more than half of all cases of MEC, and is a useful diagnostic marker $(8,9)$. The present study reports a case of MEC occurring in the sublingual gland of a 76-year-old female. A review of the relevant literature is also presented.

\section{Case report}

A 76-year old Japanese woman suffering from Parkinson's disease presented at Kurume University Hospital (Kurume, Japan) in April 2016 due to a mass in her left submandibular region. Upon examination, a soft mass was palpable. Contrast-enhanced computed tomography (CT) scans (Fig. 1A and B) and a magnetic resonance imaging 
(MRI) examination (Fig. 1C and D) revealed a cystic mass originating from the sublingual gland in the floor of the oral cavity. Fine-needle aspiration cytology was performed, which revealed only inflammatory cells, including histiocytes, lymphocytes and neutrophils, with no epithelial component. The tumor was removed percutaneously.

The sublingual gland tumor was encapsulated and did not adhere to the surrounding tissue. Macroscopically, the tumor was $20 \mathrm{~mm}$ in diameter and contained black fluid (Fig. 2). Tissue preparation and staining were performed as follows: Paraffin-embedded tissue samples were cut at $4 \mu \mathrm{m}$ and examined on a coated glass slides, and then labeled with antibodies using the BenchMark ULTRA (Ventana Automated Systems, Inc., Tucson, AZ, USA) and Bond-III autostainer (Leica Microsystems, Ltd., Milton Keynes, UK).

Primary antibodies were as follows: Endomysial antibody (EMA; cat no. 1504; ready to use; clone E29/EP1), S100 protein (cat no. N1573; ready to use) (both from DakoCytomation; Agilent Technologies, Inc., Santa Clara, CA, USA), mitochondria (cat no. 6280-0004; 1:500; clone AE1; Biogenesis; Morphosys AG, Poole, UK), p63 (cat no. M7247; 1:100, clone 4A4; DakoCytomation; Agilent Technologies, Inc.), and p40 (cat no. PC373; 1:500, clone 5-17; Calbiochem; Merck KGaA, Darmstadt, Germany).

For p63 and p40, BenchMark ULTRA was used. Briefly, each slide was heat-treated at $99^{\circ} \mathrm{C}$ using Ventana $\mathrm{CC} 1$ retrieval solution (Ventana Automated Systems, Inc.) for $30 \mathrm{~min}$, and incubated at room temperature with each antibody for $30 \mathrm{~min}$. This automated system used the streptavidin-biotin complex method with 3,3' diaminobenzidine (DAB) as the chromogen (Ventana UltraVIEW DAB detection kit; Ventana Automated Systems, Inc.). Immunostaining with EMA, S100 protein and mitochondria were performed on the same fully automated Bond-III system using on-board heat-induced antigen retrieval with epitope retrieval solution 2 ( $\mathrm{pH} 9.0$ ) for $5 \mathrm{~min}$ and a Refine polymer detection system (Leica Microsystems, Ltd.). DAB was used as the chromogen in all these immunostainings.

Histological evaluation revealed that the tumor was encapsulated by fibrous tissue (Fig. 3A) and composed of cells with clear (Fig. 3B) or granular eosinophilic cytoplasm (Fig. 3C), proliferating in solid patterns. Individual cells had subtle nuclear atypia. Hyalinized stroma was observed (Fig. 3D). A small number of mucinous cells were observed in the tumor following alcian blue (Fig. 4A) and periodic acid-Schiff (PAS) staining. Diastase-resistant PAS-positive granules were observed in the cytoplasm of the tumor cells (Fig. 4B), and were interpreted as fine hyaline globules. Immunoreactivity for p63 (Fig. 4C) and p40 (Fig. 4D) was found in the tumor cells. However, S-100 protein and epithelial membrane antigen (EMA) were not detected. A positive reaction for mitochondria was observed to be scattered in individual cells, indicating that the tumor was unlikely to be oncocytoma or oncocytic carcinoma in differential diagnosis.

Since there was no indication of perineural invasion, necrosis, mitoses or anaplasticity, the tumor was considered a low-grade malignancy according the grading scale proposed by Goode et al (10). Although microinvasion into the surrounding fibrous capsule was observed, no vascular invasion was confirmed by Elastica van Gieson and D2-40 staining.
Fluorescence in situ hybridization (FISH) analysis for a MAML2 (11q21) gene translocation was performed using a ZytoLight ${ }^{\circledR}$ SPEC MAML2 Dual Color Break Apart Probe (ZytoVision $\mathrm{GmbH}$, Bremerhaven, Germany). FISH analysis detected a split signal in the MAML2 gene, with a rate of $83 \%$ of the counted nuclei in the tumor cells (Fig. 5). Based on these findings, a final diagnosis of low-grade MEC was determined.

Following the complete surgical removal of the tumor, fludeoxyglucose-positron emission tomography (PET) revealed no other metastatic lesions. As histological evaluation demonstrated clear margins, and the MEC was determined to be a low-grade type, no additional treatment was performed. During the 6-month postoperative course, no evidence of local recurrence or distant metastasis was observed, and the patient remains relatively well thus far.

The present study was approved by the ethics committee of Kurume University (approval no. 17003) and written informed consent was obtained.

\section{Discussion}

Tumors of the sublingual gland are uncommon, and MEC of the sublingual gland is even more rare. To date, only three such cases have been reported in the English literature (11-13). In one case, bone formation was observed within the tumor (12). To the best of our knowledge, there have been no reports of MEC of the sublingual gland with confirmed MAML2 gene translocation.

MEC is typically composed of mucinous, squamoid and intermediate cells. Clear cells, oncocytic cells and columnar cells are occasionally present and prominent $(2,5)$. Since MEC exhibits various histological structures composed of these cell types, it is occasionally difficult to differentiate MEC from other types of tumor, such as clear cell, oncocytic and squamous cell carcinomas $(2,5)$.

In the present case, the tumor was composed of cells with granular eosinophilic or clear cytoplasm, and a mucinous cell component was also observed in a limited area. Therefore, it was difficult to determine a diagnosis of MEC based solely on morphological observation. Immunohistochemical staining for p40 and p63, as well as mucin staining, aided the diagnosis. Immunohistochemically, the tumor cells were negative for S-100 protein, a representative myoepithelial marker. p40 and p63 (14), which are myoepithelial as well as squamous cell markers (15), were positively expressed in the tumor cells, indicating that the tumor had features of squamous and/or squamoid differentiation. The presence of mucinous cells meant that the tumor was less likely to be an oncocytic or clear cell carcinoma. However, mucinous cells are not only detected in MEC, but also in various other tumors with mucinous metaplasia (2).

As the tumor in the present case was histologically composed of cells with granular eosinophilic or clear cytoplasm, the differential diagnoses included benign and malignant tumors with oncocytic or clear cytoplasm, such as oncocytoma, acinic cell carcinoma, mammary analogue secretory carcinoma of the salivary gland, epithelial-myoepithelial carcinoma and clear cell carcinoma. The small number of mucinous cells identified in the tumor indicated the possibility of MEC rather than other salivary gland tumors; however, 

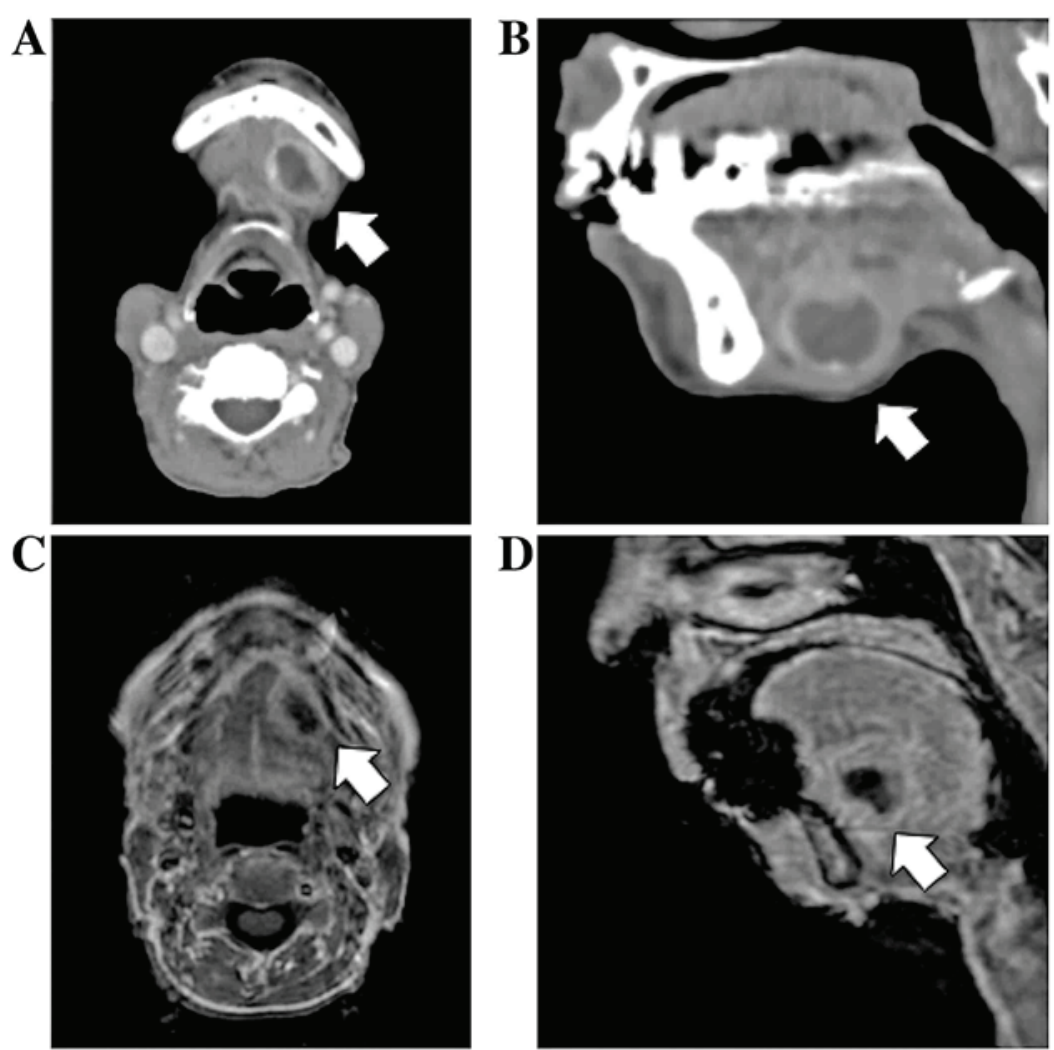

Figure 1. Imaging findings of the head and neck in a patient with sublingual gland mucoepidermoid carcinoma. (A) Axial and (B) sagittal contrast-enhanced CT scans. (C) Axial and (D) sagittal T1-weighted MRI scans. Contrast-enhanced CT and MRI scans revealed a cystic tumor lesion located in the floor of the oral cavity, growing from the sublingual gland (arrow). CT computed tomography; MRI, magnetic resonance imaging.

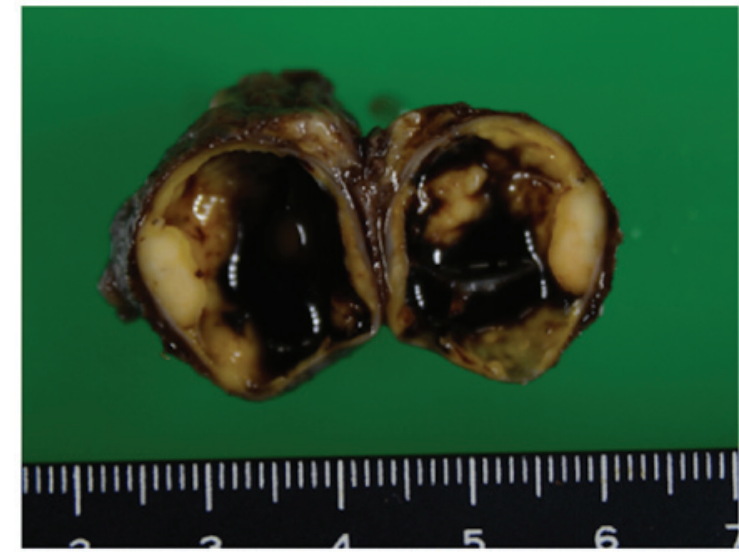

Figure 2. Macroscopic view of the tumor. The tumor was $20 \mathrm{~mm}$ in diameter and contained black fluid.

as the tumor occurred at an unual site, FISH analysis was performed to confirm this diagnosis. FISH analysis for a MAML2 gene translocation is a useful supplemental method for cases where the diagnosis of MEC is uncertain.

MAML2 gene translocation is a specific gene rearrangement present in MEC tumors, and FISH analysis of the MAML2 gene is therefore useful for the screening of MEC $(8,9)$. Noda et al $(8)$ reported that MAML2 gene translocation was detected in $\sim 65 \%$ of primary MECs, and that the cut-off value for the split signal in $M A M L$ genes is $>7 \%$. In the present case, the rate of $M A M L$ split signal detection was $83 \%$, and the tumor was therefore considered to have MAML2 gene translocation.

The majority of MECs with MAML2 gene translocation are histologically classified as low-grade (8), as demonstrated by the present case. MAML2 gene translocation is typically detected in MECs that are predominantly composed of epidermoid, intermediate and mucinous cells; however, there have been a few reports of clear cell or oncocytic variants of MEC harboring a $M A M L 2$ gene split $(16,17)$.

The MAML2 gene translocation is an oncogenic event that underlies the development of MEC (18). Therefore, MAML2 gene translocation may be observed in various types of cells in MEC. Tumor cells with a granular eosinophilic or clear cytoplasm may indicate oncogenesis as the degree of MAML2 gene translocation is high in the tumor.

Although FISH analysis for a MAML2 gene translocation is not routinely performed to diagnose MEC, its use in unusual cases may aid in determining an accurate diagnosis. The MAML2 gene and other molecular biomarkers have been identified to form the basis for the development of novel therapeutic strategies (19). Noda et al (8) reported that MAML2 gene translocation is associated with a favorable prognosis in cases of MEC. However, a more recent study reported that MAML2 gene status has no association with the prognosis of MEC (20). As the association between MAML2 status and prognosis is controversial, MAML2 status in MEC must be investigated in a larger cohort of patients in order to determine its precise roles. MAML2 gene translocation may also aid the prediction of prognoses and selection of optimal treatments. 

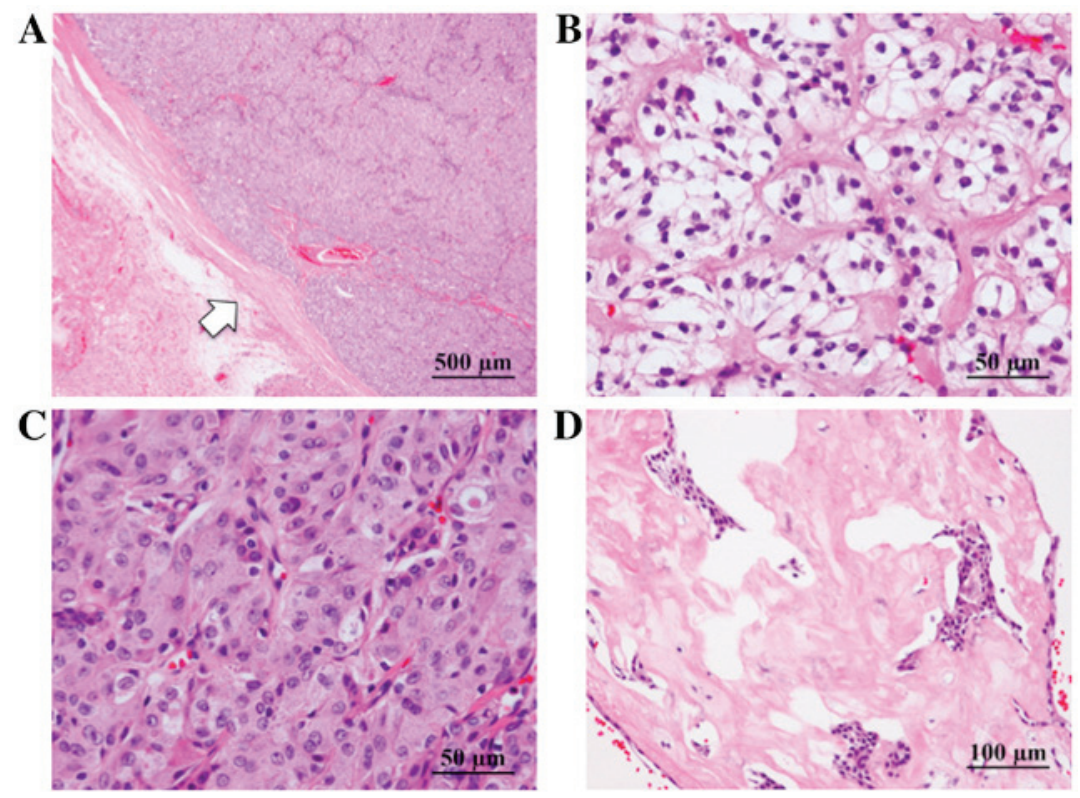

Figure 3. Pathological findings of the tumor following hematoxylin and eosin staining. (A) The tumor was encapsulated by fibrous tissue (arrow). (B-D) The tumor was composed of cells with low-grade nuclear atypia, with (B) clear or (C) granular eosinophilic cytoplasm; a solid pattern of cell proliferation was observed, along with (D) hyalinized stroma.
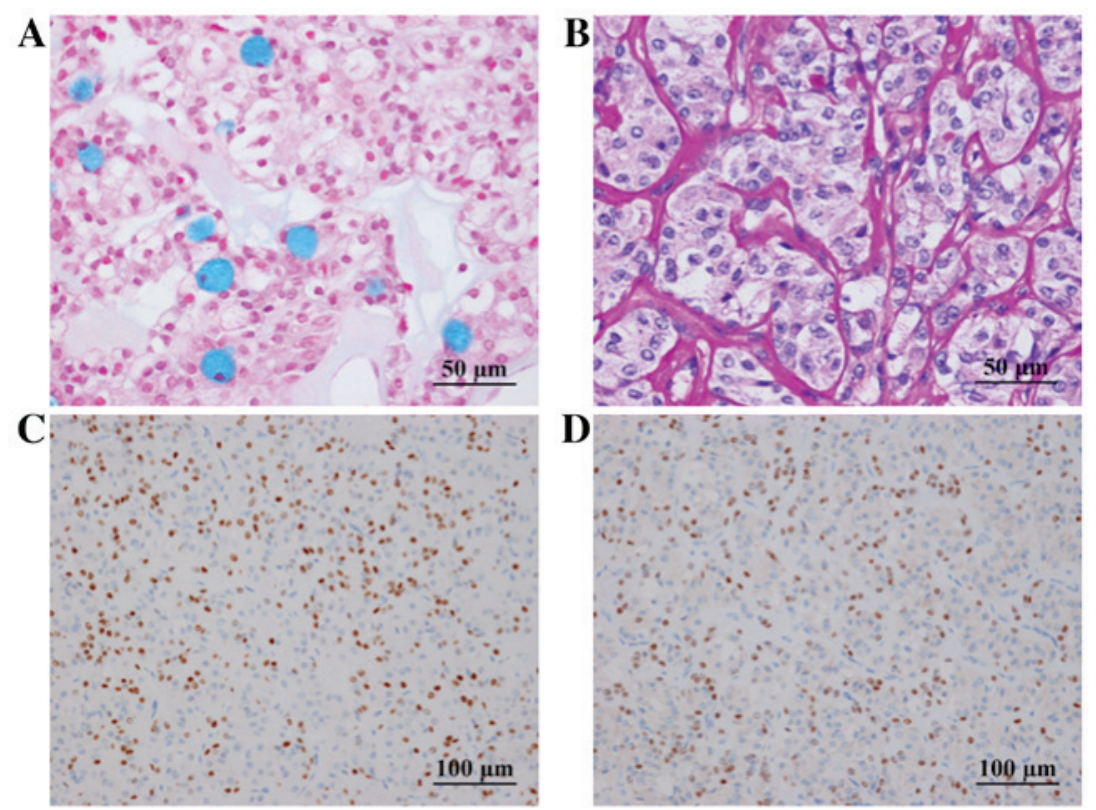

Figure 4. (A) Alcian blue staining revealed mucinous cells in the tumor. (B) Diastase-digestion PAS staining revealed diastase-resistant PAS-positive globules . The cells exhibited positive immunoreactivity for (C) p63 and (D) p40. PAS, periodic acid-Schiff.
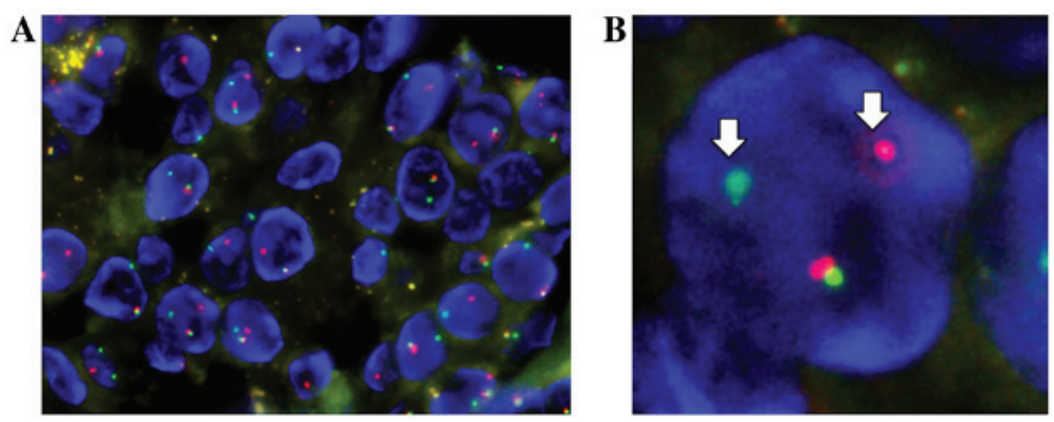

Figure 5. Fluorescence in situ hybridization analysis of MAML2 gene translocation. (A) The split signals of the $M A M L 2$ gene were present in $83 \%$ of the counted nuclei in tumor cells. (B) Arrows indicates the split signals (one green and one red) of tumor cells. MAML2, mastermind-like transcriptional coactivator 2. 
The 5-year survival rates of low-grade and high-grade MEC are 97 and 27\%, respectively (21). Therefore, it is important to determine the histological grade of this malignancy, as this influences the clinical features and prognosis (22). MEC may be classified into three grades according to its pathological features, including cystic components, perineural invasion, necrosis, mitoses and anaplasticity $(2,3,5,10)$. As the tumor in the present case lacked any of these features, it was categorized as a low-grade malignancy.

Surgical resection of the primary tumor is the standard treatment for all grades of MEC. The majority of cases of high-grade MEC tend to metastasize, and selective neck dissection during initial surgery is therefore recommended (22). In addition, adjuvant radiotherapy is recommended in cases of high-grade MEC (22). By contrast, low-grade MEC is less aggressive; thus, surgical resection alone is usually sufficient, as in the present case. However, there is a potential risk of local metastasis in low-grade MEC, and this occurs in $\sim 4 \%$ of cases (10). Therefore, careful clinical follow-up is recommended.

Previous reports have described the utility of PET/CT for MECs of the lung or bronchi $(23,24)$, and one report described the assessment of low-grade MEC with high-resolution $\mathrm{PET} / \mathrm{CT}$ (24). It is unclear whether FDG accumulates in low-grade MEC. However, we suggest that PET/CT combined with CT and MRI could be used as a supplemental examination, as the prognosis and the frequency of metastasis in MECs of the sublingual gland are unclear. The precise roles of PET/CT for salivary gland MECs must be investigated in a larger patient cohort.

In summary, the combined use of immunohistochemistry, FISH analysis of MAML2 gene translocation and histological observation is a useful and reliable examination technique for the accurate diagnosis of atypical MEC. To the best of our knowledge, this is the first report of MEC of the sublingual gland with MAML2 gene translocation confirmed by FISH.

\section{References}

1. Eveson JW and Cawson RA: Salivary gland tumours. A review of 2410 cases with particular reference to histological types, site, age and sex distribution. J Pathol 146: 51-58, 1985.

2. Gary LE and Paul LA: Tumor of the salivary glands. AFIP Atlas of Tumor Pathology Series 4. AFIP ARP, Washington, pp173-196, 2008.

3. Goode RK and El-Naggar AK: Mucoepidermoid carcinoma. In: World Health Organization Classification of Tumors. Pathology and Genetics of Head and Neck Tumors. Barnes L, Evenson JW, Reichart P and Sidransky D (eds). IARC Press, Lyon, pp219-220, 2005.

4. Stewart FW, Foote FW and Becker WF: Muco-epidermoid tumors of salivary glands. Am J Surg 122: 820-844, 1945.

5. John WE and Lester DRT: Malignant neoplasms of the salivary glands. In: Head and Neck Pathology. Lester DRT (ed). 2nd edition. Elsevier Sauners, Philadelphia, pp270-277, 2013.

6. Luna MA: Salivary mucoepidermoid carcinoma: Revisited. Adv Anat Pathol 13: 293-307, 2006.
7. Whatley WS, Thompson JW and Rao B: Salivary gland tumors in survivors of childhood cancer. Otolaryngol Head Neck Surg 134: 385-388, 2006.

8. Noda H, Okamura Y, Nakayama T, Miyabe S, Fujiyoshi Y, Hattori H, Shimozato K and Inagaki H: Clinicopathological significance of MAML2 gene split in mucoepidermoid carcinoma. Cancer Sci 104: 85-92, 2013.

9. Fehr A, Röser K, Heidorn K, Hallas C, Löning T and Bullerdiek J: A new type of MAMAL2 fusion in mucoepidermoid carcinoma. Genes Chromosomes Cancer 47: 203-206, 2008.

10. Goode RK, Alclair PL and Ellis GL: Mucoepidermoid carcinoma of the major salivary glands. Clinical and histopathologic analysis of 234 cases with evaluation of grading criteria. Cancer 82: 1217-1224, 1998

11. Kumar AN, Nair PP, Thomas S, Raman PS and Bhambal A: Mucoepidemoid carcinoma of sublingual gland: A malignant neoplasm in an uncommon region. BMJ Case Rep May 2011: bcr0220113864, 2011.

12. Murase Y, Kawano S, Kiyoshima T, Goto Y, Matsubara R, Chikui T, Yoshiga D and Nakamura S: Case of mucoepidermoid carcinoma of the sublingual gland accompanied with extensive dystrophic calcification and intratumoral bone formation. Head Neck 37: E161-E164, 2015.

13. Sumanth KN, Mainali A, Ongole R and Pai MR: Mucoepidermoid carcinoma: 'A Mimicker'? J Nepal Dent Assoc 10: 31-34, 2009.

14. Furuse C, Sousa SO, Nunes FD, Magalhães MH and Araújo VC: Myoepithelial cell markers in salivary gland neoplasms. Int $\mathbf{J}$ Surg Pathol 13: 57-65, 2005.

15. Owosho AA, Aguilar CE and Seethala RR: Comparison of p63 and p40 $(\Delta \mathrm{Np} 63)$ as basal, squamoid, and myoepithelial markers in salivary gland tumors. Appl Immunohistochem Mol Morphol 24: 501-508, 2016.

16. Tajima S, Namiki I and Koda K: A clear cell variant of mucoepidermoid carcinoma harboring CRTC1-MAML2 fusion gene found in buccal mucosa: Report of a case showing a large clear cell component and lacking typical epidermoid cells and intermediate cells. Med Mol Morphol 50: 117-121, 2017.

17. Fujimaki M, Fukumura Y, Saito T, Mitani K, Uchida S, Yokoyama J, Yao T and Ikeda K: Oncocytic mucoepidermoid carcinoma of the parotid gland with CRTC1-MAML2 fusion transcript: Report of a case with review of literature. Hum Pathol 42: 2052-2055, 2011.

18. Wu L, Liu J, Gao P, Nakamura M, Cao Y, Shen H and Griffin JD: Transforming activity of MECT1-MAML2 fusion oncoprotein is mediated by constitutive CREB activation. EMBO $\mathrm{J} 24$ : 2391-2402, 2005.

19. Stenman G, Persson F and Andersson MK: Diagnostic and therapeutic implications of new molecular biomarkers in salivary gland cancers. Oral Oncol 50: 683-690, 2014.

20. Seethala RR and Chiosea SI: MAML2 status in mucoepidermoid carcinoma can no longer be considered a prognostic marker. Am J Surg Pathol 40: 1151-1153, 2016.

21. Spiro RH, Huvos AG, Berk R and Strong EW: Mucoepidermoid carcinoma of salivary gland origin. A clinicopathologic study of 367 cases. Am J Surg 136: 461-468, 1978.

22. Nance MA, Seethala RR, Wang Y, Chiosea SI, Myers EN, Johnson JT and Lai SY: Treatment and survival outcomes based on histologic grading in patients with head and neck mucoepidermoid carcinoma. Cancer 113: 2082-2089, 2008.

23. Elnayal A, Moran CA, Fox PS, Mawlawi O, Swisher SG and Marom EM: Primary salivary gland-type lung cancer: Imaging and clinical predictors of outcome. AJR Am J Roentgenol 201: W57-W63, 2013.

24. Ishizumi T, Tateishi U, Watanabe S, Maeda T and Arai Y: F-18 FDG PET/CT imaging of low-grade mucoepidermoid carcinoma of the bronchus. Ann Nucl Med 21: 299-302, 2007. 